Revue des patrimoines et plasticiens (XXe-XXle siècles)

\title{
De la revue d'avant-garde à la reconnaissance publique : la collaboration entre architectes et plasticiens à Liège dans les années 1930
}

From avant-garde review to public recognition; the collaboration between architects and artists in Liege during the 1930s

Sébastien Charlier

\section{OpenEdition}

Journals

Édition électronique

URL : http://journals.openedition.org/insitu/15061

DOI : 10.4000/insitu.15061

ISSN : 1630-7305

Éditeur

Ministère de la culture

Référence électronique

Sébastien Charlier, «De la revue d'avant-garde à la reconnaissance publique : la collaboration entre architectes et plasticiens à Liège dans les années 1930 », In Situ [En ligne], 32 | 2017, mis en ligne le 19 juillet 2017, consulté le 03 mai 2019. URL : http://journals.openedition.org/insitu/15061 ; DOI: 10.4000/insitu.15061

Ce document a été généré automatiquement le 3 mai 2019.

\section{c) (†)}

In Situ Revues des patrimoines est mis à disposition selon les termes de la licence Creative Commons Attribution - Pas d'Utilisation Commerciale - Pas de Modification 4.0 International. 


\section{De la revue d'avant-garde à la reconnaissance publique : la collaboration entre architectes et plasticiens à Liège dans les années 1930}

From avant-garde review to public recognition; the collaboration between architects and artists in Liege during the 1930s

\section{Sébastien Charlier}

1 La collaboration entre les architectes et les plasticiens à Liège dans l'entre-deux-guerres trouve l'une de ses racines les plus importantes dans l'avant-garde de la fin du XIXe siècle. Le développement de l'architecture moderne à travers le courant de l'Art nouveau donne aux architectes et aux plasticiens un cadre de réflexion qui leur permet d'échanger et d'envisager des collaborations très étroites. En 1895, l'architecte et décorateur Gustave Serrurier-Bovy (1858-1910) crée L'CEuvre artistique ${ }^{1}$ qui offre aux architectes et artistes liégeois un environnement propice à l'émulation et aux échanges. Ce petit cercle d'acteurs engagés dans la modernité gravite autour de l'imprimeur d'origine française Auguste Bénard (1854-1907) chez qui sculpteurs, architectes, peintres se rencontrent, confrontent leurs conceptions et envisagent leurs collaborations. De la fin du xix siècle au début de la Première Guerre mondiale, cette pluridisciplinarité se concrétise principalement dans l'habitation unifamiliale, les pouvoirs publics locaux se montrant particulièrement rétifs aux idées nouvelles. L'intervention des plasticiens se concentre en façade par le biais de la sculpture décorative dont Oscar Berchmans (1869-1950) apparaît comme l'un des représentants les plus prolifiques. Ses œuvres se retrouvent dans de nombreuses réalisations d'architectes engagés dans la modernité comme Paul Jaspar (1859-1945), Victor Rogister (1874-1955) ou Joseph Nusbaum (1876-1950). Des collaborations plus fortes s'expriment également par d'autres voies. La transformation de la maison de l'architecte Paul Comblen (1869-1955) voit ainsi des interventions qui 
touchent tant l'intérieur que l'extérieur de la construction. On retrouve la signature du peintre Jacques Jacobi (1877-1957) dans la grande toile placée dans la cage d'escalier ainsi que dans l'ensemble des vitraux, et celle d'Oscar Berchmans notamment dans le sgraffite en façade et dans les bas-reliefs en plâtre qui décorent le salon. Mis à part l'une ou l'autre réalisation, la collaboration entre architectes et plasticiens est discrète au lendemain de la Première Guerre mondiale. Les cercles d'avant-garde créés à la fin du XIX ${ }^{e}$ siècle ont disparu et c'est dans le monde des petites revues qu'il faut aller chercher les premiers signes d'un nouvel élan.

\section{Les premières collaborations : le rôle des revues}

Dans le courant des années 1920, Liège se tient dans l'ombre de Bruxelles. Son engagement dans le bouillonnement artistique qui touche la Belgique est modeste mais il est loin d'être anodin. Se distinguant par sa précarité, le circuit local de l'avant-garde se compose de personnalités qui s'empressent de quitter le pays. Le poète Paul Dermée (1886-1951), le musicien Arthur Pétronio (1897-1983) ou le graveur Marcel LempereurHaut (1898-1986) s'en vont participer respectivement à la fondation des revues L'Esprit nouveau (Paris), Créer (Reims) et Vouloir (Lille). Dans ce contexte, l'émergence de la figure de Georges Linze (1900-1993), instituteur et écrivain, apparaît comme exemplaire. Marqué par les théories futuristes développées par Marinetti dès 1909, Linze s'entoure de poètes, graveurs et dessinateurs locaux. En décembre 1920, avec Marcel Lempereur-Haut et René Liège (1900-1998), il fonde le Groupe moderne d'art de Liège qui, en janvier 1921, publie son manifeste ${ }^{2}$. En mars 1921 paraît le premier numéro d'Anthologie, la revue du "Groupe moderne d'art de Liège ». Dans son introduction, l'organe met en avant son objectif de vulgarisation et de diffusion de l'art moderne: "Un de ses devoirs est de présenter et d'expliquer au grand public la manière des artistes nouveaux, quelles que soient leurs tendances $\rrbracket^{3}$. Elle s'intéresse aux grands courants littéraires et artistiques de l'entre-deux-guerres (dadaïsme, constructivisme, surréalisme, unanimisme, etc.) avec une prédilection pour le futurisme: la machine, la vitesse, l'usine et la jeunesse sont des images récurrentes. Cheville ouvrière de la revue, Linze s'inscrit d'emblée dans une vision transversale de la littérature: "Tout est poétisé ou poétisable ${ }^{4}$ ", déclare-t-il en décembre 1921 (fig. 1). 


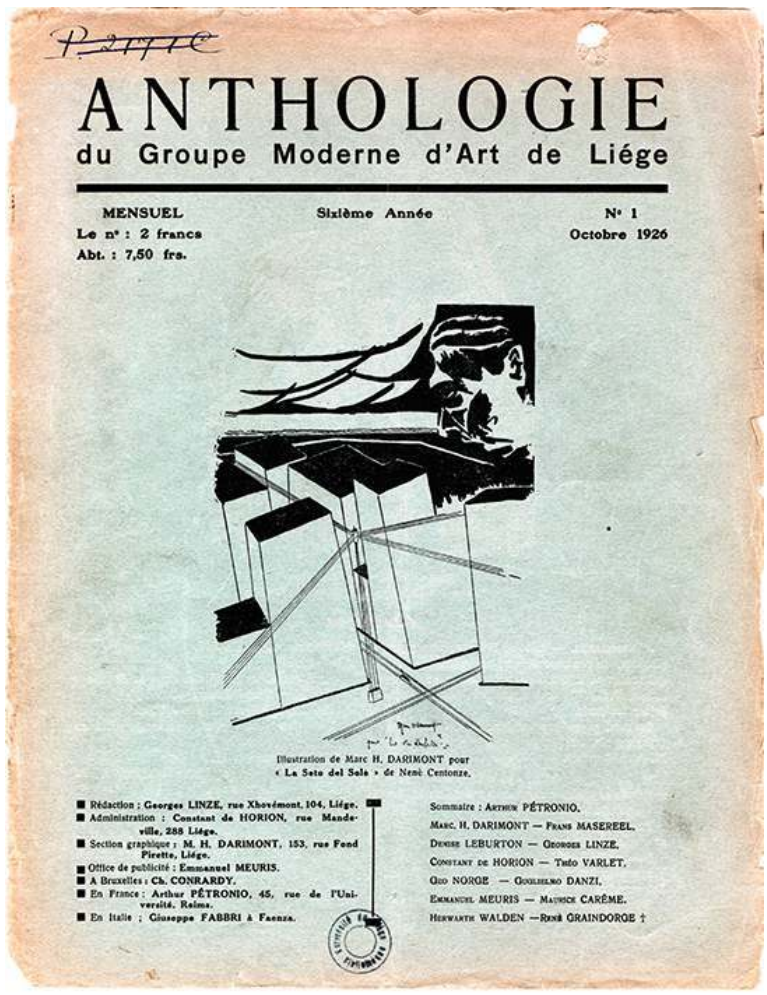

Darimont Marc H., La sete del sole. Couverture de la revue Anthologie, octobre 1926, n¹.

Anthologie témoigne d'une foi sans limites dans un monde nouveau et réclame la nécessaire adaptation de l'art à la civilisation moderne dans une perspective rationaliste : «il est incontestable que l'industrialisation moderne, les découvertes scientifiques surtout dans les domaines considérés jusqu'à ce jour comme hermétiques, modifient notre genre de vie et par conséquent notre façon de penser $»^{5}$. Pour Linze, l'artiste joue un rôle de « réceptacle » et doit formaliser la civilisation moderne. Cet art moderne est le fruit de son époque et Linze, rempli d'optimisme, y décrit une société nouvelle marquée par les transformations issues de la Première Guerre mondiale et les progrès tant technologiques (découvertes scientifiques) que culturels (sports, « interpénétration des races ») et sociaux (élargissement du droit de vote, etc.).

Pour Linze, l'art doit quitter les cénacles intellectuels pour toucher toutes les couches de la population car «la masse est isolée de l'avant-garde $»^{6}$. La vulgarisation du propos constitue ainsi un enjeu majeur en vue d'imposer l'art moderne dans la société belge. Loin d'être singulière, cette pensée s'inscrit dans une réflexion sur l'art social dont Victor Bourgeois est une des grandes figures en Belgique. Son article «Le beau par l'utile» publié en 1925 dans la revue du Parti ouvrier belge (РОВ) L'Avenir social ${ }^{7}$ marque d'ailleurs profondément Linze qui en reproduit de nombreux extraits. Sur le terrain local, Anthologie apparait comme un fabuleux porte-voix des artistes liégeois. Elle publie notamment les travaux du graveur Marcel H. Darimont (1903- ?), des peintres Fernand Steven (1895-1955) et Edmond Delsa (1875-1957) ou du sculpteur Idel Ianchelevici (1904-1994).

5 Bien que peu abordée dans la revue, l'architecture est une matière qui suscite la curiosité de Linze. En 1928, le poète se rapproche de quelques étudiants de l'académie des Beaux- 
Arts de Liège qui viennent de fonder L'Équerre, une revue entièrement dédiée à l'architecture et à l'urbanisme. Fortement influencés par la revue 7 Arts, les jeunes Liégeois, parmi lesquels figurent Yvon Falise (1908-1981) et Jean Moutschen (1907-1965), en reprennent les idéaux, le ton et surtout l'idée d'une révolution en marche basée sur une conception pluridisciplinaire de la pratique de l'architecture. Cette pensée ne peut que séduire Georges Linze. Outre l'édition d'une revue qui se poursuivra jusqu'en 1939 (fig. 2), L'Équerre mène diverses opérations de propagande censées sensibiliser les étudiants de l'académie à l'art et à l'architecture moderne.

Figure 2

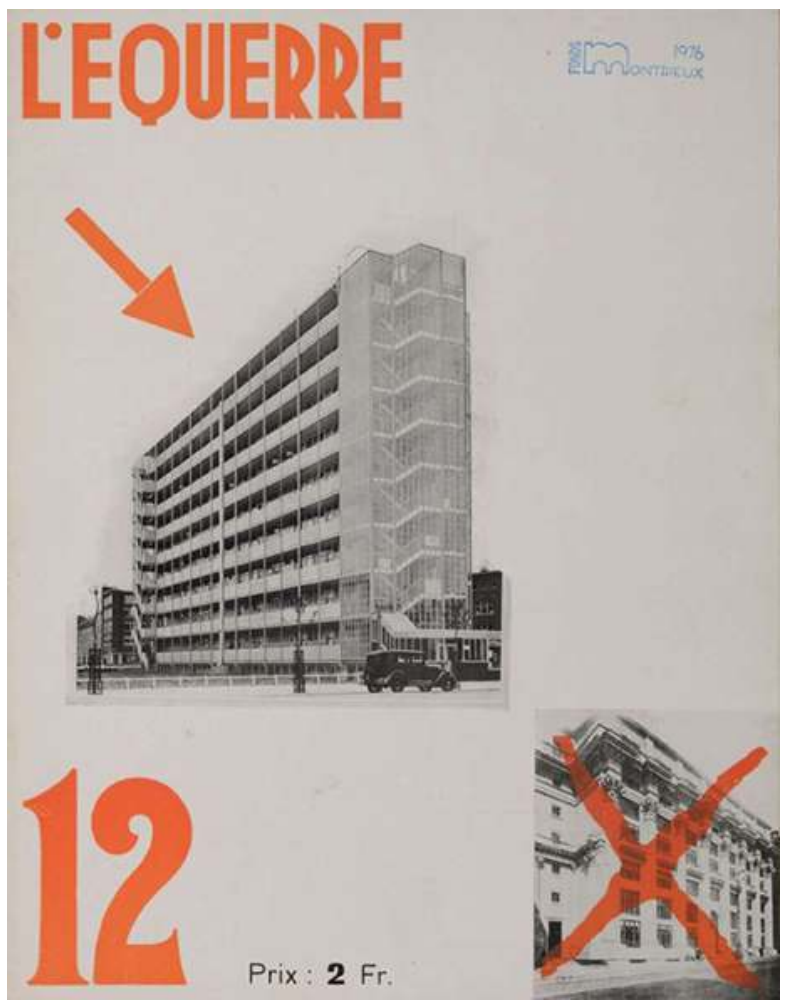

Couverture de la revue L'Équerre, décembre $1934, n^{\circ} 12$.

Le 26 novembre 1929, Georges Linze tient à l'académie une conférence sur "L'art moderne et la Wallonie $»^{8}$ dans laquelle il porte un regard très critique sur sa ville :

Tout, dit le conférencier, nous montre une ville aux traditions enracinées et dorlotées par une bourgeoisie de mauvais goût: rappelons-nous cette burlesque histoire du mur de la place Saint-Lambert; ce beffroi proposé place du Marché où ces pauvres styles, déjà malmenés, devaient venir s'entasser les uns sur les autres. Admirons dans le passé ce qui est beau, nous en avons des exemples, mais que les cadavres des vieilles pierres ne viennent pas encombrer notre chemin ${ }^{9}$.

7 Cette intervention inaugure une série de textes dans lesquels Linze met à mort le XIX siècle: "Le XIX siècle, tout le monde sait cela, a couvert l'Europe de monuments baroques, débauche de pierres et de styles ; sans atteindre à autre chose qu'au comique et à la boursouflure. L'architecture la plus ridicule est bien celle du Palais de Justice de Bruxelles $»^{10}$. Le poète pointe la machine et la jeunesse comme les nouveaux vecteurs d'une création digne $\mathrm{du} \mathrm{xx}^{\mathrm{e}}$ siècle. La conférence et les écrits qui l'accompagnent marquent le début d'une longue et fructueuse collaboration entre Anthologie et L'Équerre, 
Linze se chargeant dès décembre 1929 de la direction artistique de la revue d'architecture. L'Équerre ouvre alors ses pages à la peinture, la littérature, la musique (essentiellement le jazz) et au cinéma. Grâce à lui, les jeunes rédacteurs perçoivent les rapports qui unissent l'architecture aux autres expressions artistiques contemporaines. Les liens avec Anthologie se resserrent progressivement et les échanges d'articles ou d'illustrations sont nombreux. L'Équerre publie les œuvres d'artistes liégeois comme Fernand Steven ou Marcel H. Darimont. Quant à la poésie, elle s'impose peu à peu avec, outre les contributions de Linze, des textes de Maurice Carême (1899-1978) et de Marcel Loumaye (1889-1956). Si L'Équerre voit son champ s'ouvrir, elle n'en reste pas moins une revue d'architecture. C'est peu après avoir reçu Georges Linze qu'elle se rapproche d'une autre figure de l'avant-garde belge. Le 4 janvier 1930, Victor Bourgeois est invité, probablement par l'entremise de Linze, à présenter une conférence consacrée à "L'architecture moderne ${ }^{11}$. Structuré en trois parties et accompagné d'une projection de photographies, l'exposé traite d'abord des origines de l'architecture moderne, puis des questions d'urbanisme et enfin présente quelques œuvres de l'auteur.

8 Les liens privilégiés entre les architectes liégeois et Georges Linze vont permettre l'émergence à Liège d'un mouvement d'avant-garde certes tardif, mais d'une grande vitalité. Les échanges ne se limitent pas à des complicités éditoriales mais s'ouvrent également aux événements destinés à un public plus large. Les deux expositions organisées en commun ne cessent de gagner en visibilité. Intitulée «Exposition d'architecture rationnelle et éléments ", la première manifestation se tient en 1932 dans les locaux du peintre Joseph Koenig (1878-1971). Si l'événement est couvert par quelques journalistes locaux, il reste confidentiel, alors que l'exposition «Pour une meilleure architecture » qui se tient en 1933 au palais des Beaux-Arts de Liège est, quant à elle, plus ambitieuse. Chaque groupe y dispose de son propre espace (fig. 3) mais les échanges entre artistes et architectes s'élaborent, en particulier dans la maison-type réalisée par les architectes du groupe L'Équerre et décorée notamment par Idel Ianchelevici (1909-1994) et Fernand Steven (fig. 4). Pour la première fois depuis la guerre, une grande exposition montre aux yeux des Liégeois et de leurs mandataires politiques un front uni d'architectes et d'artistes de l'avant-garde. 
Figure 3

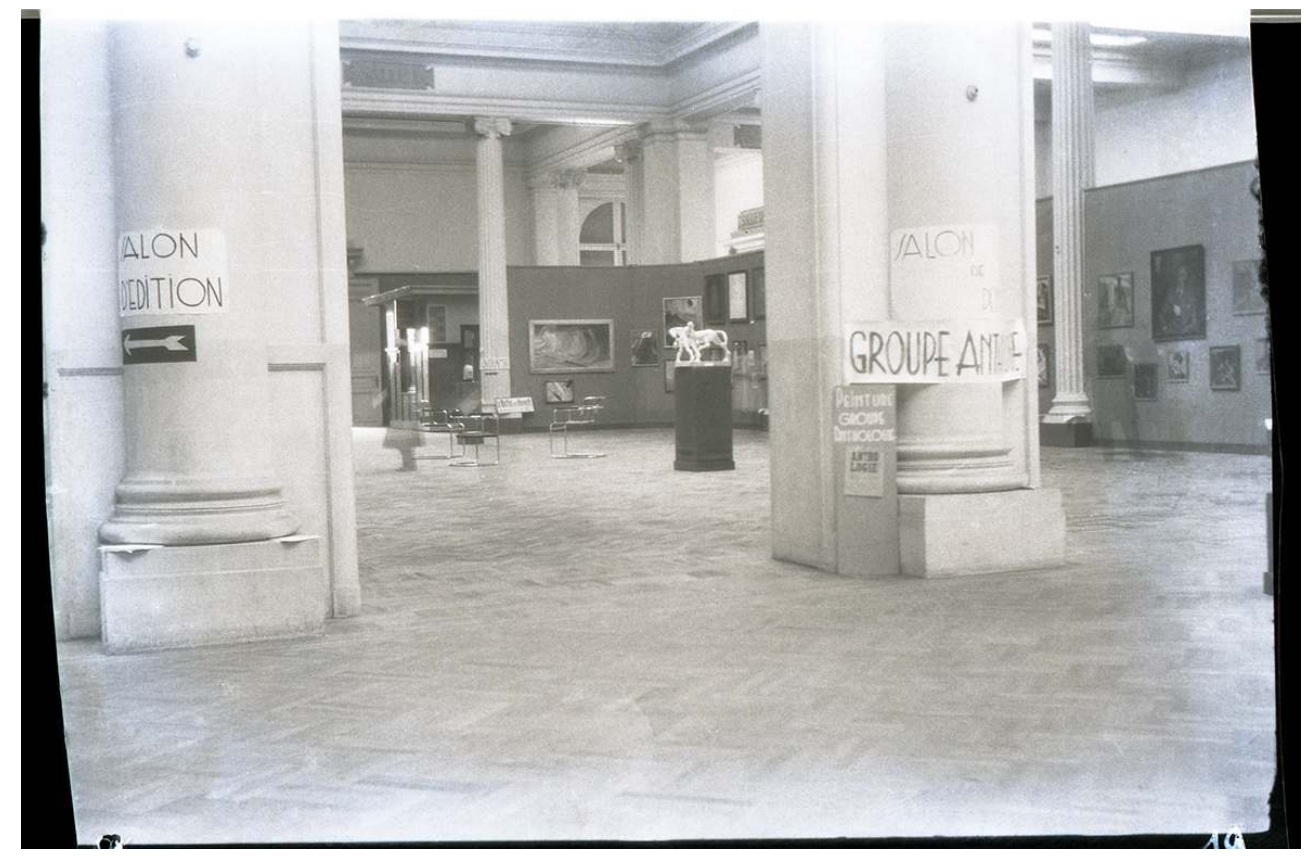

Exposition « Pour une meilleure architecture ». Photographie de la section réservée à la revue Anthologie, palais des Beaux-Arts de Liège, mars 1933.

(c) Collection privée. 


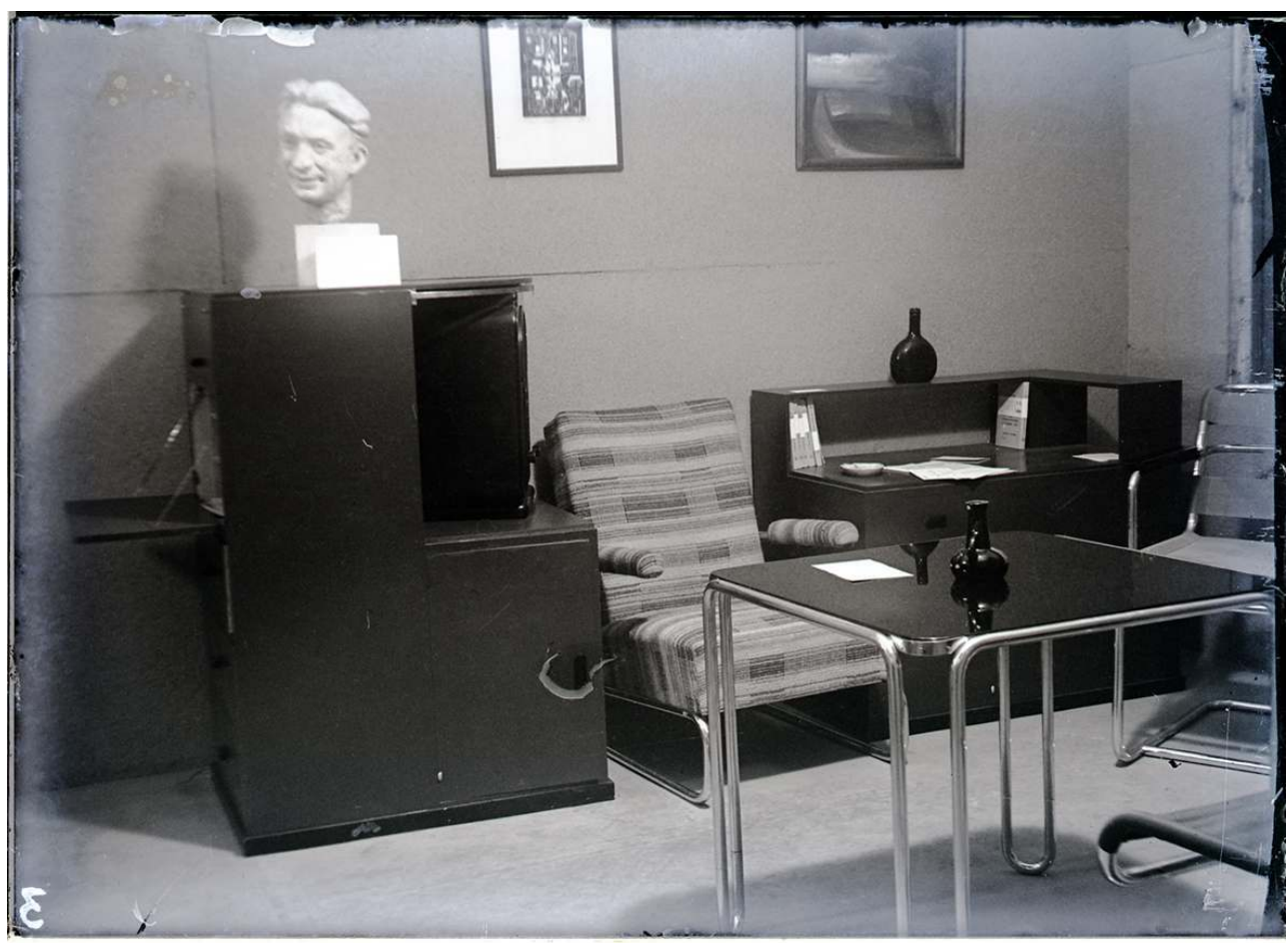

Exposition «Pour une meilleure architecture ». Photographie de la tête sculptée par Idel lanchelevici accompagnée de la toile de Fernand Steven installées dans la salle à manger de la maison-type réalisée par les architectes de L'Équerre, avec la participation de Louis Herman de Koninck, palais des Beaux-Arts de Liège, mars 1933.

(c) Collection privée.

\section{Concrétiser les projets : le soutien des pouvoirs publics}

9 Si les collaborations entre architectes et artistes de l'avant-garde sont intenses, elles dépassent rarement le cadre des expositions ou des revues. La crise économique qui touche durement la société belge écarte les artistes de la commande. Il faut attendre la seconde moitié des années 1930 pour voir se constituer un contexte politique favorable permettant la réalisation concrète des idéaux de L'Équerre et d'Anthologie. En 1935, Paul Van Zeeland (1893-1973) prend la tête d'une coalition tripartite associant le parti catholique, le parti libéral et le POB. Le gouvernement met en place différentes mesures afin de lutter contre la crise et instaure notamment une politique de grands travaux. Elle crée ainsi l'office du redressement économique (OREC), sorte de bras armé du programme de relance ${ }^{12}$. La nouvelle institution se présente comme une incroyable opportunité pour certains, grâce à la modernisation de l'État et de ses infrastructures en particulier, qui répond parfaitement aux aspirations humanistes et collectivistes proclamées, affichées, revendiquées par les architectes belges du Mouvement moderne. La direction artistique de l'OREC est en outre confiée à Henry van de Velde (1863-1957) qui valide la cohérence architecturale des projets soumis. Aux quatre coins du pays naît ainsi une architecture « officielle » socialiste. 
10 À Liège, les choses évoluent également favorablement. En 1935, la nouvelle coalition communale voit les socialistes disposer de l'échevinat des travaux publics qui est confié à Georges Truffaut (1901-1942). La modernisation des bâtiments publics va connaître un formidable coup d'accélérateur. Les écoles, les aires de jeux ou les infrastructures sportives se multiplient dans la région liégeoise. Jean Moutschen (1907-1965), l'un des fondateurs de la revue L'Équerre, entré au service communal de l'architecture en 1933, se voit confier des projets d'envergure dont le lycée Léonie de Waha constitue certainement l'œuvre la plus aboutie. Les origines de l'établissement scolaire remontent à 1868, date de la création par la baronne Léonie de Waha de Chestret (1836-1926) d'un "institut supérieur libre de Demoiselles» permettant à certaines jeunes filles d'accéder à l'enseignement secondaire ${ }^{13}$. En 1925, l'école prend le titre de «lycée » et conduit aux études universitaires. Très vite, à l'étroit dans ses locaux de la rue des Célestines, elle nécessite de nouvelles installations. La Ville envisage d'abord l'agrandissement du bâtiment de la rue des Célestines puis, en 1932, décide de construire un complexe sur des terrains lui appartenant situés à l'angle des rues Jonfosse et Pouplin ${ }^{14}$. En 1933, les premiers plans sont adoptés par le conseil communal ${ }^{15}$. En 1936, la Ville, qui n'a pas encore entamé les travaux, revoit ses plans et décide de construire sur un terrain plus grand et surtout plus proche du centre, sur le boulevard d'Avroy ${ }^{16}$. Le programme est ambitieux : le bâtiment doit rassembler vingt-sept classes, deux gymnases, une piscine, une salle de spectacle de 900 places, un internat de quatre-vingt-quinze chambres ainsi qu'un abri antiaérien ${ }^{17}$ (fig. 5).

Figure 5

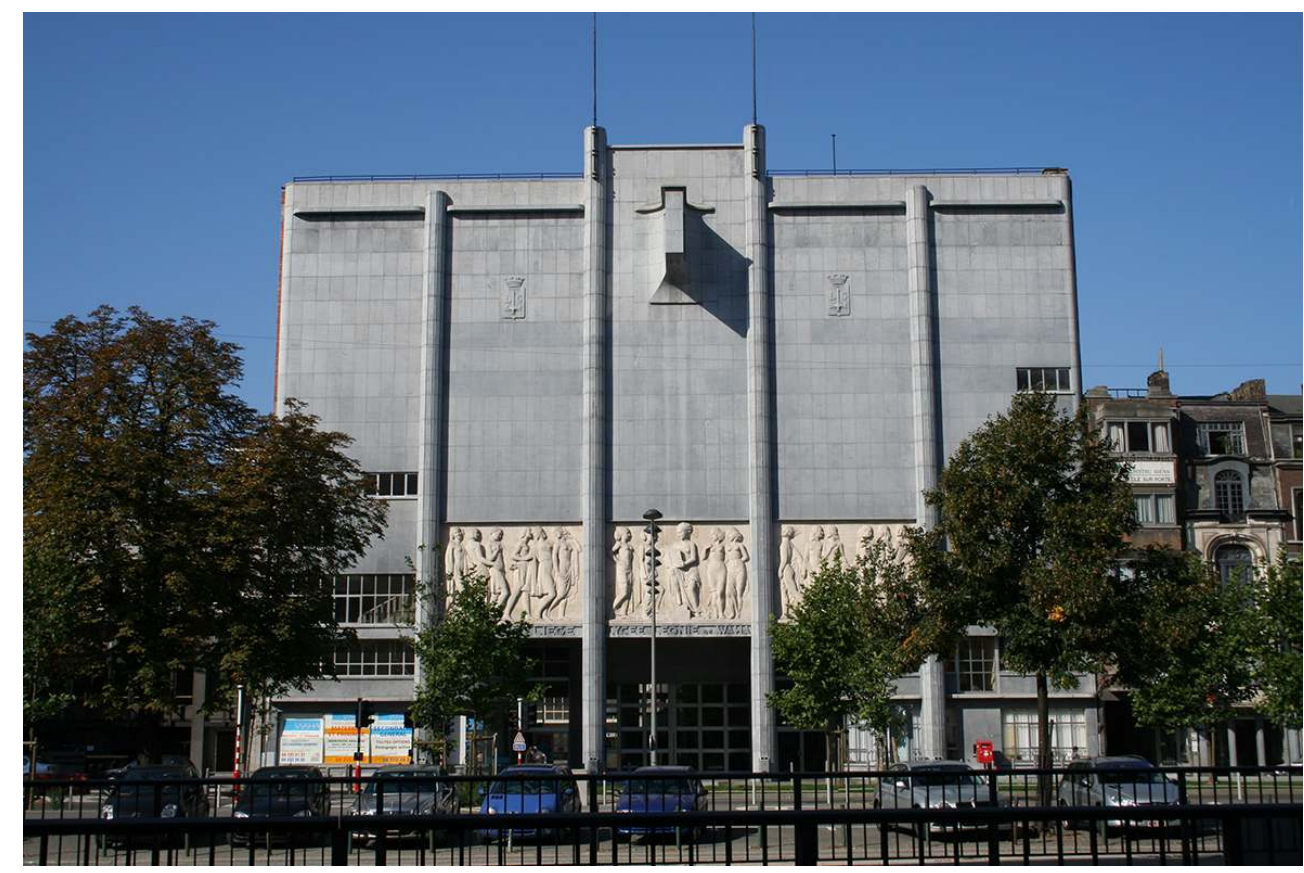

Lycée Léonie de Waha, arch. Jean Moutschen, 1936-1938. Photographie de la façade principale. Phot. Charlier, Sébastien, 2013. (c) Sébastien Charlier.

La parcelle est relativement étroite mais se développe généreusement en profondeur. Le complexe se compose de quatre immeubles qui ceinturent la cour de récréation. Le bâtiment principal abrite le hall et la grande salle de spectacle. Orientée à l'est et ne nécessitant pas d'être éclairée naturellement, la salle s'emboîte dans une construction 
dont les murs sont aveugles. Son emplacement à front de voirie permet par ailleurs d'isoler les classes du tumulte de la rue. Se développant en profondeur, le bâtiment en épi est idéalement orienté au sud. Les trois premiers niveaux accueillent les classes tandis que les deux derniers abritent l'internat avec, au $4^{\mathrm{e}}$ étage, quatre-vingt-dix chambres séparées par des cloisons légères et, au $5^{\mathrm{e}}$, le réfectoire, la cuisine, la salle de jeux, la salle de musique, les locaux de la direction. Au sud, une longue galerie-promenade donne accès aux salles d'étude et sert aussi d'espace de détente. Pour favoriser l'apport en lumière naturelle, Moutschen réalise une façade entièrement vitrée et équipée de hauts châssis métalliques. Sur la toiture-terrasse, l'architecte dispose un solarium réservé aux enfants de l'internat. Le troisième bâtiment en L ferme la cour et abrite les deux salles de gymnastique et la piscine. Au-delà du souci de fonctionnalité s'impose celui de la qualité esthétique. Les jeunes filles doivent évoluer dans un cadre propice à l'apprentissage où l'art occupe une place centrale. Confiée à l'architecte mais certainement encadrée par les autorités politiques, la commande artistique est très ouverte. Le choix des intervenants donne d'ailleurs l'impression d'un consensus puisqu'il associe grandes figures académiques et représentants de l'avant-garde. En cette période de crise, c'est l'ensemble des artistes qui doit être soutenu. De même, s'agissant d'une vitrine exceptionnelle pour la Ville de Liège, il convient de présenter la pluralité des tendances qui composent le paysage culturel local.

12 Ainsi, les interventions à l'extérieur du bâtiment se distinguent par une écriture classique, en particulier pour ce qui est des bas-reliefs situés sur la façade principale. Monumentale, celle-ci s'insère en bas d'un immense rectangle ( 30 x 31 mètres) aveugle presque entièrement recouvert de dalles de petit granit. Traitées en frise, les œuvres de Louis Dupont (1896-1967), Adelin Salle (1884-1952) et Robert Massart (1892-1955) mettent en scène un groupe d'adolescentes drapées à l'antique (fig. 6), reposant sur de robustes colonnes qui se prolongent sur toute la hauteur de l'édifice. 
Figure 6

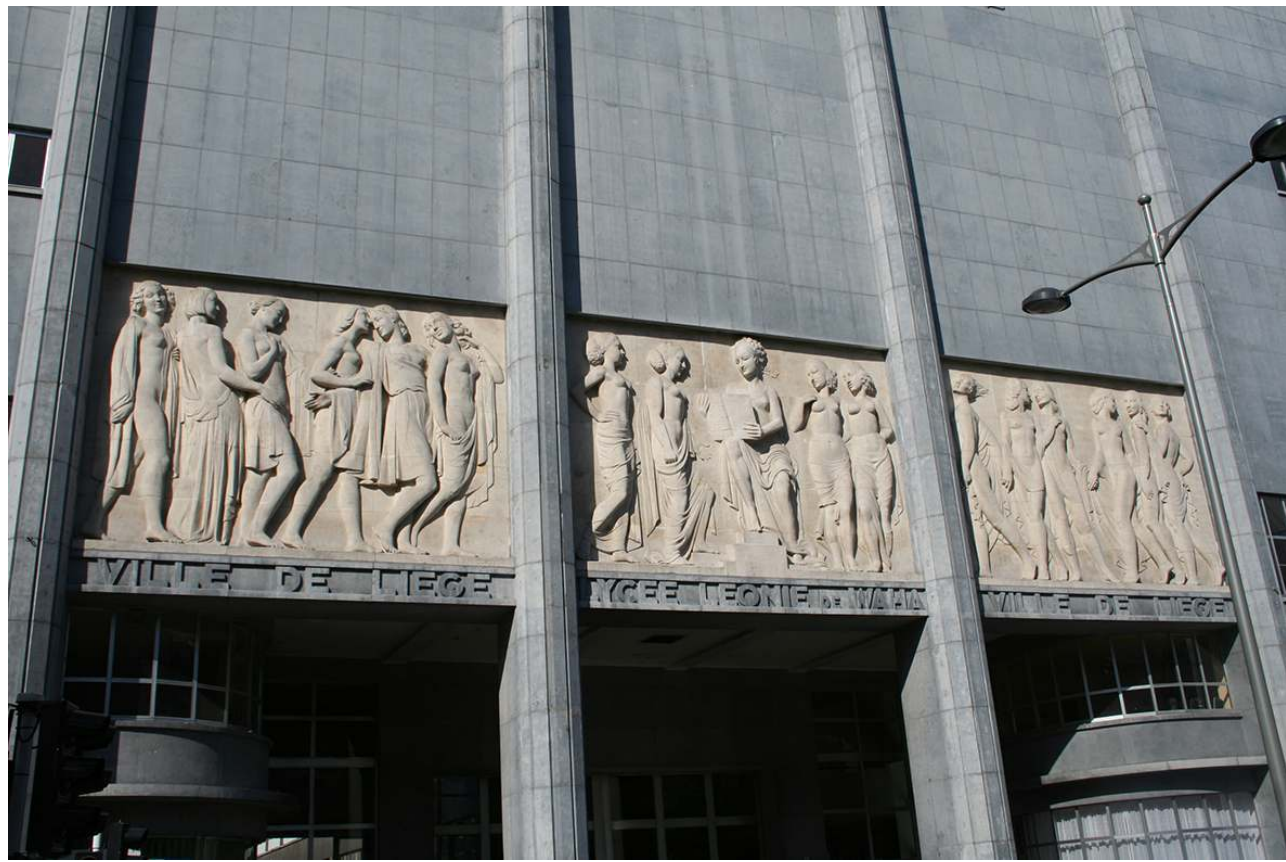

Lycée Léonie de Waha, arch. Jean Moutschen, 1936-1938. Photographie de la composition de Louis Dupont, Adelin Salle et Robert Massart sur la façade principale.

Phot. Charlier, Sébastien, 2006. ( ) Sébastien Charlier.

Dans cette école pour jeunes filles, les thèmes de l'apprentissage se conjuguent aussi autour de la féminité. Oscar Berchmans (1869-1950) s'essaie à une nouvelle pratique artistique. La mosaïque monumentale installée sur la façade arrière du bâtiment principal témoigne d'une parfaite maîtrise de la technique (fig. 7). 


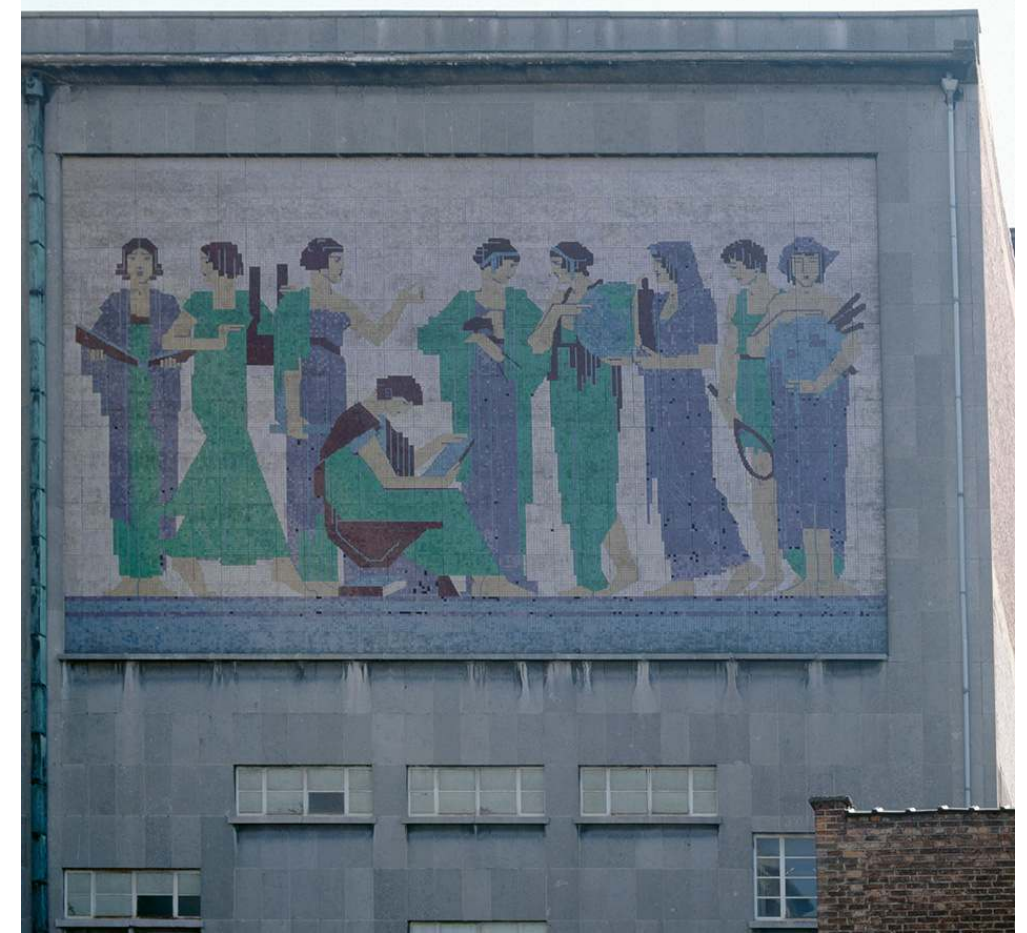

Lycée Léonie de Waha, arch. Jean Moutschen, 1936-1938. Photographie de la mosaïque monumentale par Oscar Berchmans sur la façade arrière.

Phot. Focant, Guy, 1999. ( ) SPW-Patrimoine, photographie n E66.95.902.

Elle représente dans une grande simplification formelle neuf figures féminines drapées portant divers éléments symbolisant l'enseignement du sport, de la littérature, de la science et des arts (raquette de tennis, livre, globe terrestre, lyre, palette, etc.). D'autres interventions visent à célébrer la puissance économique ou les beautés naturelles de la région. Dans le petit hall qui conduit à la cour de récréation, les toiles de Marcel Jaspar (1886-1952) montrent un paysage industriel ainsi qu'une scène champêtre. La bibliothèque est quant à elle décorée d'un panneau de Joseph Verhaeghe (1900-1987) représentant des usines à Ougrée et un bas-relief de Louis Gérardy (1887-1959) illustrant le travail à la mine. Edmond Delsa (1875-1957), proche collaborateur des revues Anthologie et L'Équerre, peint une vue de Huy qui est installée dans la salle d'étude du deuxième étage. Parmi ces interventions relativement conventionnelles, d'autres tendances plus proches de l'avant-garde se retrouvent également. Jean Moutschen confie à Fernand Steven (1895-1955) la décoration de la salle de physique et du laboratoire de chimie. Le peintre, proche collaborateur de Georges Linze, du «Groupe d'art moderne de Liège » et de L'Équerre, réalise deux toiles abstraites de tendance futuriste aux lignes très nerveuses (fig. 8). 
Figure 8

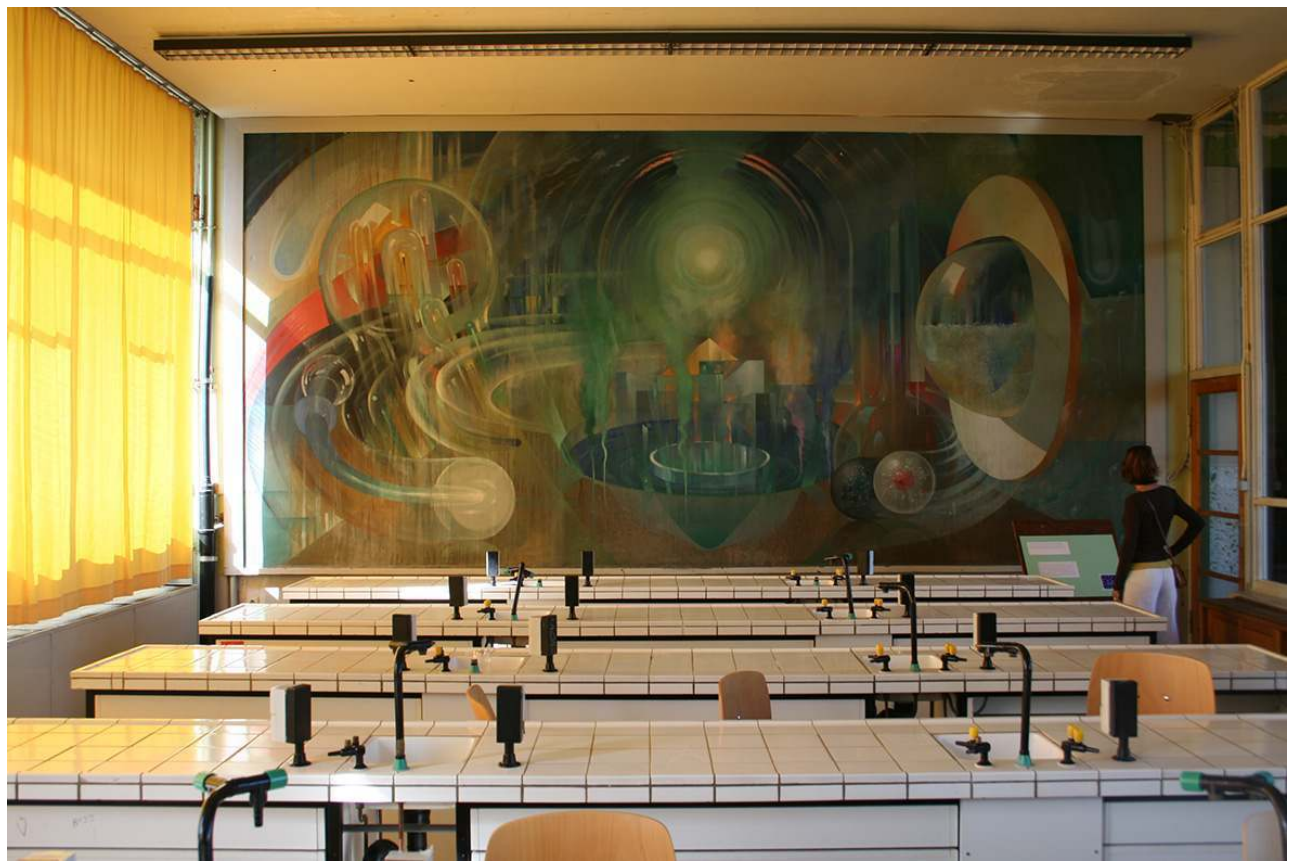

Lycée Léonie de Waha, arch. Jean Moutschen, 1936-1938. Photographie de la composition de Fernand Steven dans la classe de chimie.

Phot. Charlier, Sébastien, 2006. ( ) Sébastien Charlier.

Dans la grande salle des fêtes, ce sont des compositions expressionnistes qui ceinturent l'espace. Le progrès de l'Humanité grâce à l'étude, œuvre de Robert Crommelynck (1895-1968) (fig. 9) fait face à un travail plus classique d'Auguste Mambour (1896-1968) (fig. 10) montrant des personnages prenant diverses poses. 


\section{Figure 9}

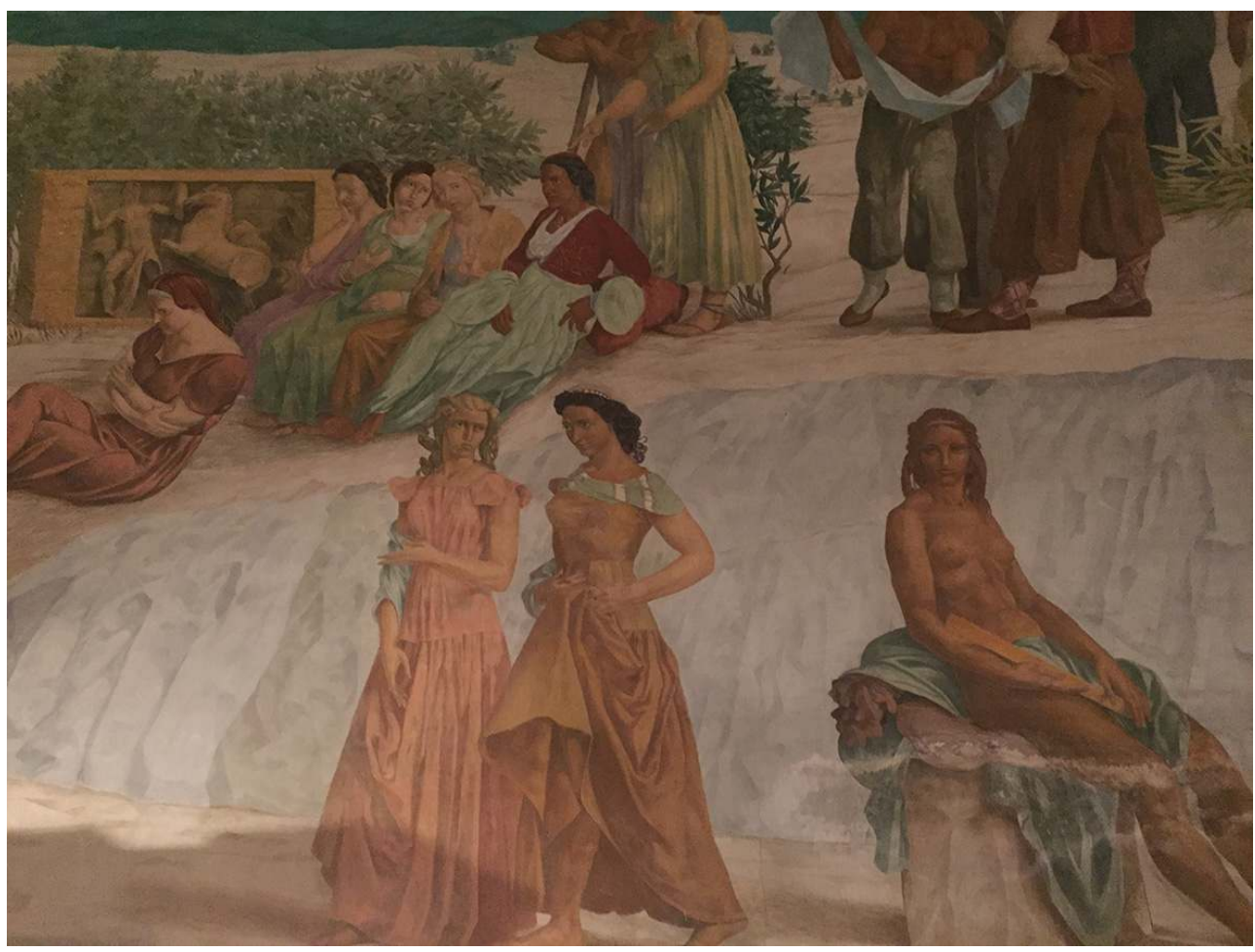

Lycée Léonie de Waha, arch. Jean Moutschen, 1936-1938. Photographie d'un détail de la composition de Robert Crommelynck intitulée Le progrès de l'Humanité grâce à l'étude et située dans la salle de spectacle.

Phot. Charlier, Sébastien, 2015. (c) Sébastien Charlier. 


\section{Figure 10}

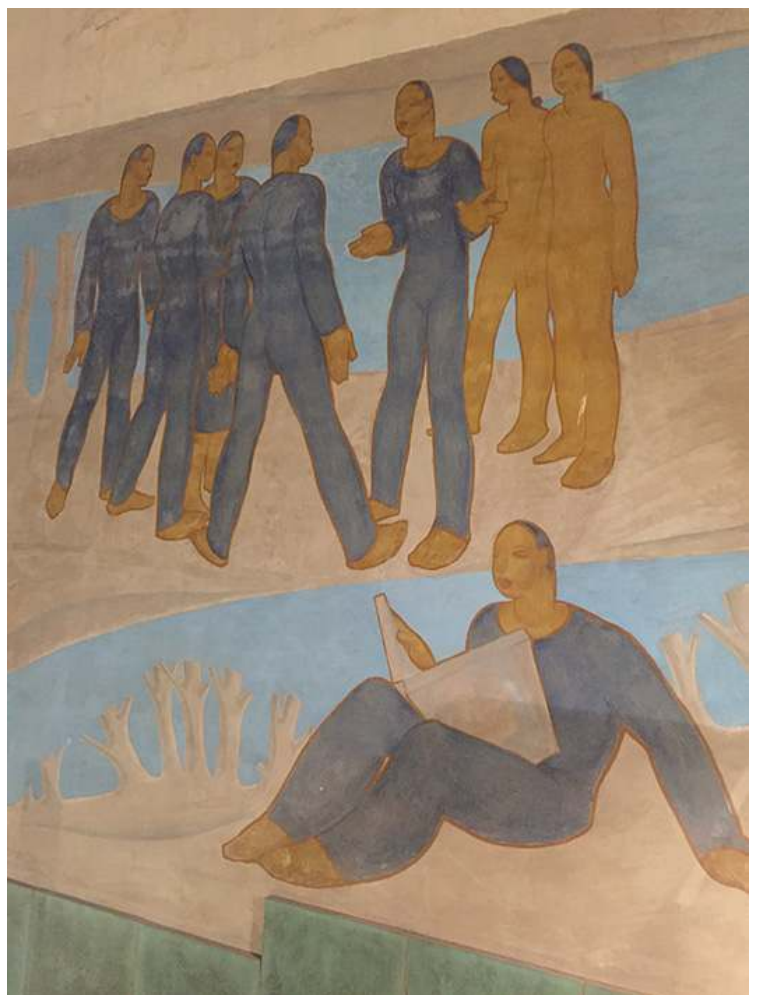

Lycée Léonie de Waha, arch. Jean Moutschen, 1936-1938. Photographie de la composition d'Auguste Mambour dans la salle de spectacle.

Phot. Charlier, Sébastien, 2015. (c) Sébastien Charlier.

De l'autre côté de la cour de récréation, la piscine réunit deux autres artistes proches de l'avant-garde. Marcel Caron (1890-1961) dessine une série de vitraux qui mettent en scène une régate d'aviron sous les yeux de la foule (fig. 11). 


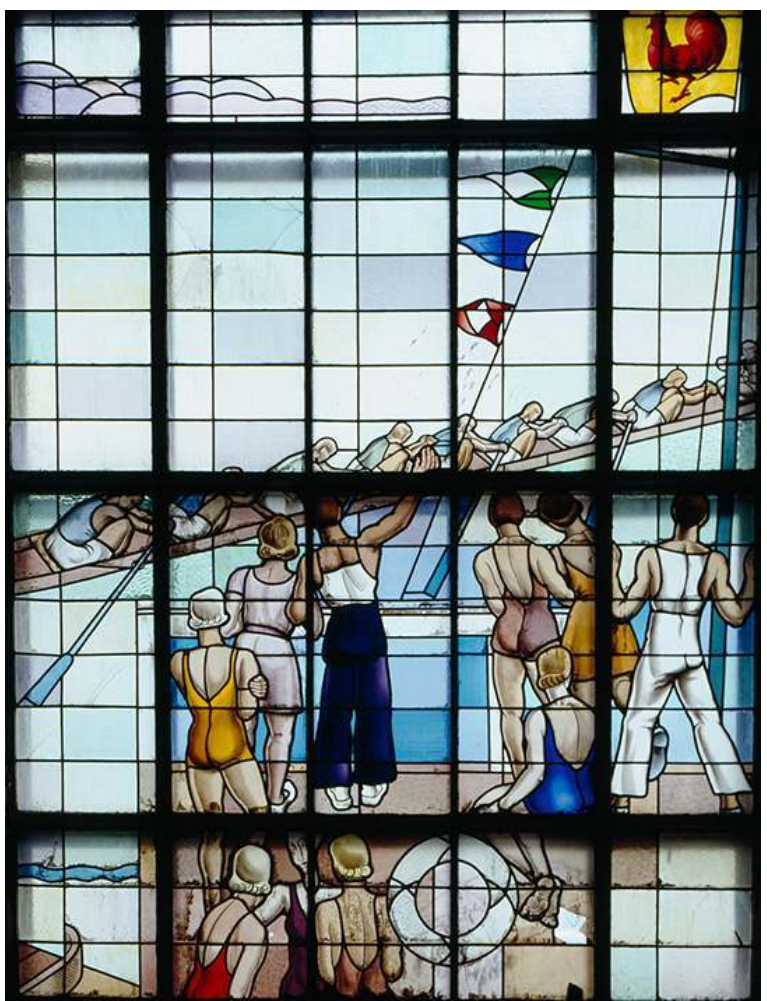

Lycée Léonie de Waha, arch. Jean Moutschen, 1936-1938. Photographie du vitrail réalisé par Marcel Caron et installé dans le bâtiment de la piscine.

Phot. Focant, Guy, 1999. (c) SPW-Patrimoine, photographie nE45.04.081

En face, de l'autre côté du bassin, Adrien Dupagne (1889-1980) réalise six panneaux en mosaïque figurant des fonds marins exotiques. Sur un mur, une mosaïque représente les différents mouvements de la brasse (fig. 12). Une fois encore, l'esthétique est mise au service de la pédagogie tout en utilisant une thématique très liée à l'enfance. En effet, le lycée se distingue moins par le choix des artistes que par la situation des œuvres dans ses bâtiments. Si ces derniers réunissent des interventions monumentales, de nombreuses œuvres sont disposées à proximité immédiate des élèves. Qu'il s'agisse des toiles de Stevens ou des reliefs de jeunes filles réalisés par Georges Petit (1879-1958) et situés dans le hall principal, l'art se rapproche de son public. Le processus de décloisonnement des arts trouve ici une expression manifeste tout en réalisant les ambitions sociales poursuivies par les auteurs d'Anthologie et de L'Équerre. L'intérêt que porte Jean Moutschen à l'intégration des œuvres d'art ne se limite pas au lycée Léonie de Waha. Le statut des plasticiens, et particulièrement leur position dans l'architecture publique, se voit ainsi confortée et appuyée par l'architecte qui déclare en 1938 vouloir consacrer cinq pour cent du budget à la décoration des bâtiments communaux ${ }^{18}$. 
Figure 12

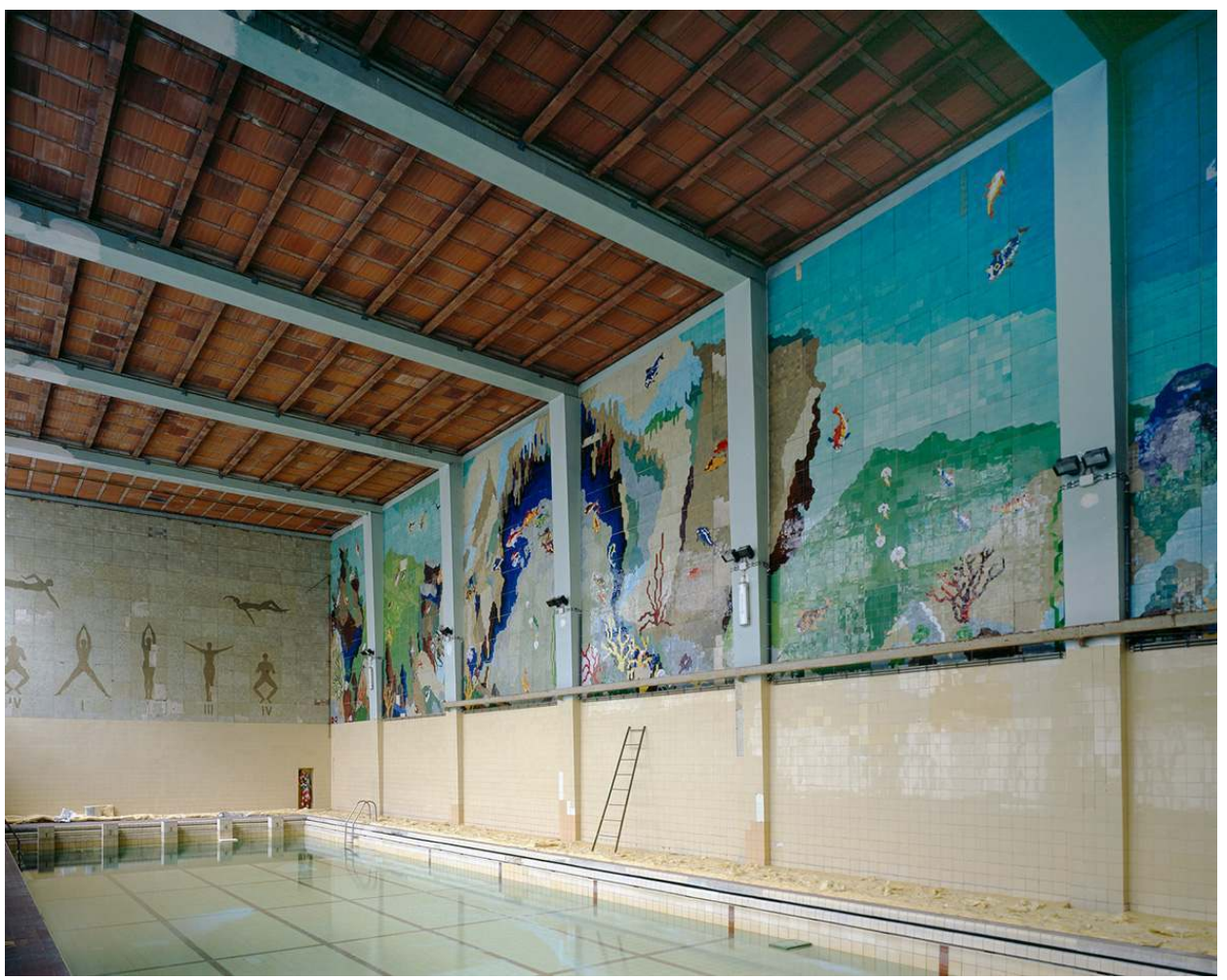

Lycée Léonie de Waha, arch. Jean Moutschen, 1936-1938. Photographie de la composition en mosaïque réalisée par Adrien Dupagne et installée dans le bâtiment de la piscine.

Phot. Focant, Guy, 1999. (c) SPW-Patrimoine, photographie n E45.04.084.

Dans le cadre de l'Exposition internationale de l'eau qui doit se tenir en 1939, la construction du palais des fêtes, dessiné en 1937 par Jean Moutschen, fait à nouveau appel à des artistes locaux. Le bâtiment principal est précédé d'un grand hall entièrement vitré et surmonté d'un bas-relief. CEuvre d'Adolphe Wansart (1873-1954), il est composé de figures représentant l'industrie (mineur, chimiste, armurier) et les arts (architecte, écrivain, sculpteur, peintre et musicien) autour d'un personnage féminin, allégorie de la Ville de Liège ${ }^{19}$ (fig. 13). 


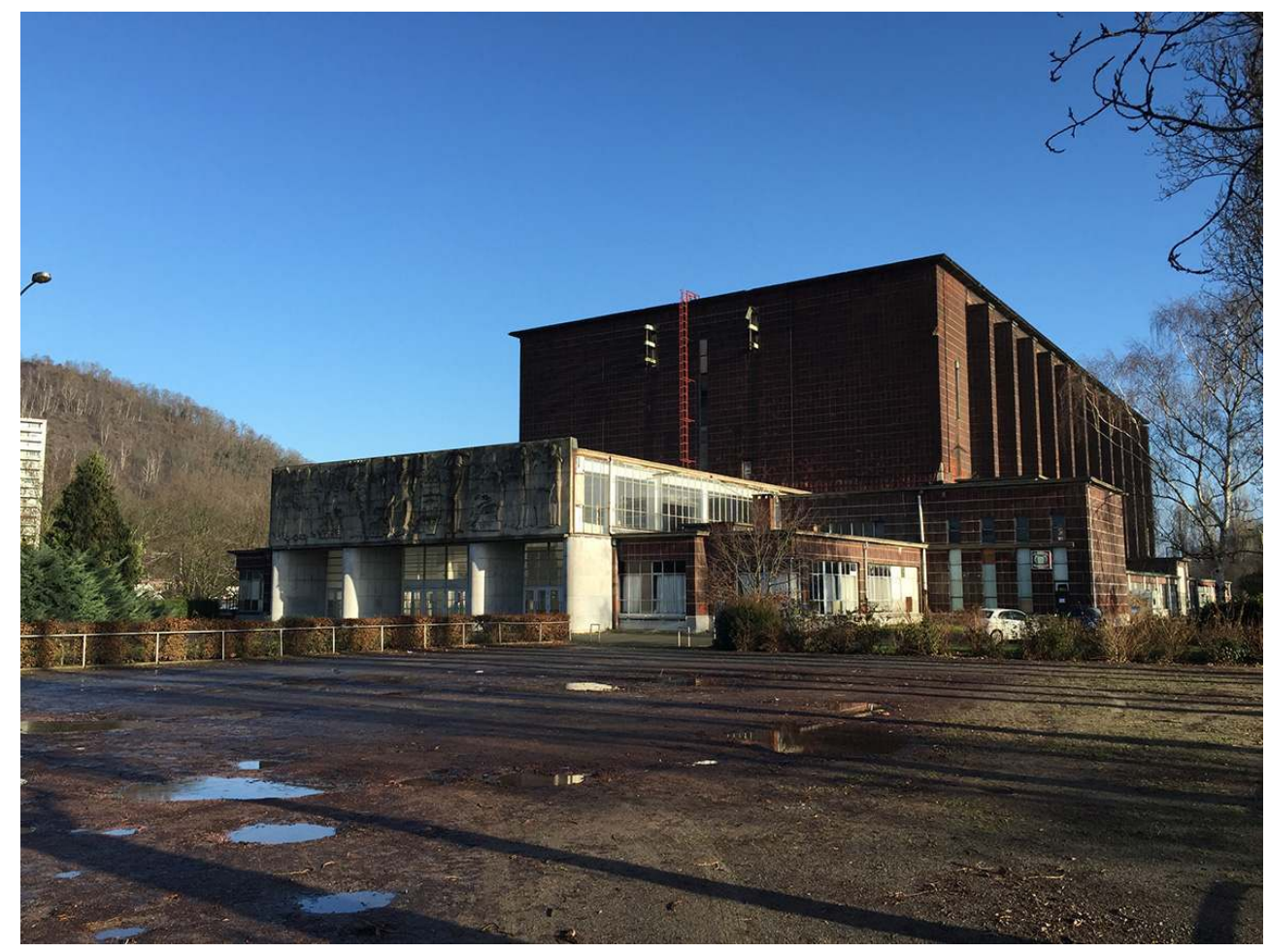

Palais permanent de la Ville de Liège, ancienne patinoire, arch. Jean Moutschen, 1938-1939. Photographie de la façade principale.

Phot. Charlier, Sébastien, 2014. @ Sébastien Charlier.

La clinique de la Société mutuelle des administrations publiques (SMAP) réalisée par Joseph Moutschen (1895-1977), également proche de la revue L'Équerre, voit plusieurs artistes collaborer à nouveau (fig. 14). L'architecte, comme les administrateurs de la SMAP, accorde une grande importance à l'environnement des patients : "Ce n'était pas assez de donner au blessé des soins corporels, il fallait aussi le placer dans un milieu aussi agréable et aussi confortable que possible $\aleph^{20}$. Les matériaux utilisés pour les revêtements intérieurs répondent à des questions d'hygiène et témoignent d'un budget généreux : escaliers en marbre, carrelages aux joints dorés, portes avec carreaux biseautés, etc. Joseph Moutschen fait appel à plusieurs artistes pour décorer les espaces. On retrouve ainsi les interventions d'auteurs qui avaient œuvré dans le lycée Léonie de Waha: une peinture murale de Fernand Steven, un bas-relief de Louis Dupont et des vitraux ornant les trois œils-de-bœuf par Edgar Scauflaire (1893-1960) (fig. 15). Les compositions de Robert Crommelynck et de Jean Donnay (1897-1992) ont, quant à elles, disparu. 
Figure 14

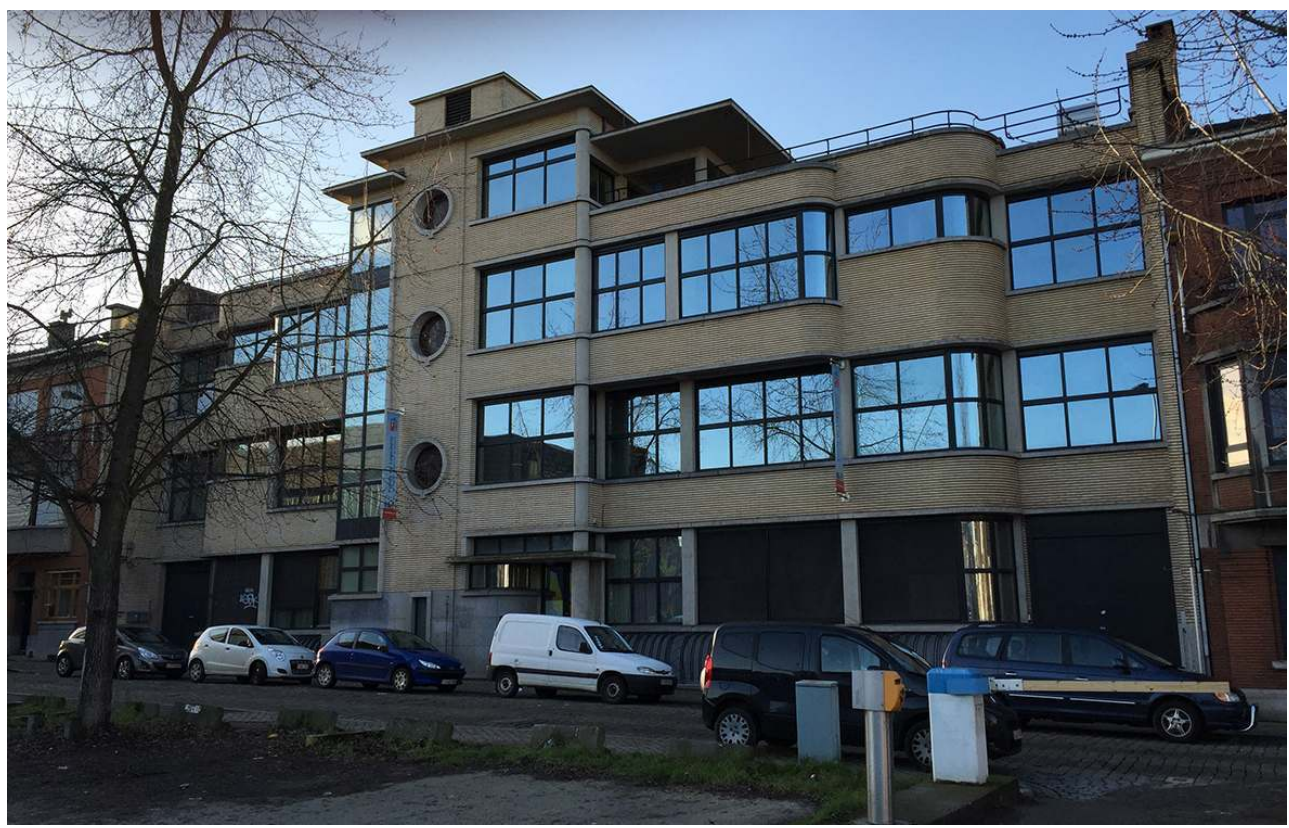

Clinique Jules Seeliger, arch. Joseph Moutschen, 1938-1948. Photographie de la façade principale. Phot. Charlier, Sébastien, 2015. @ Sébastien Charlier.

Figure 15

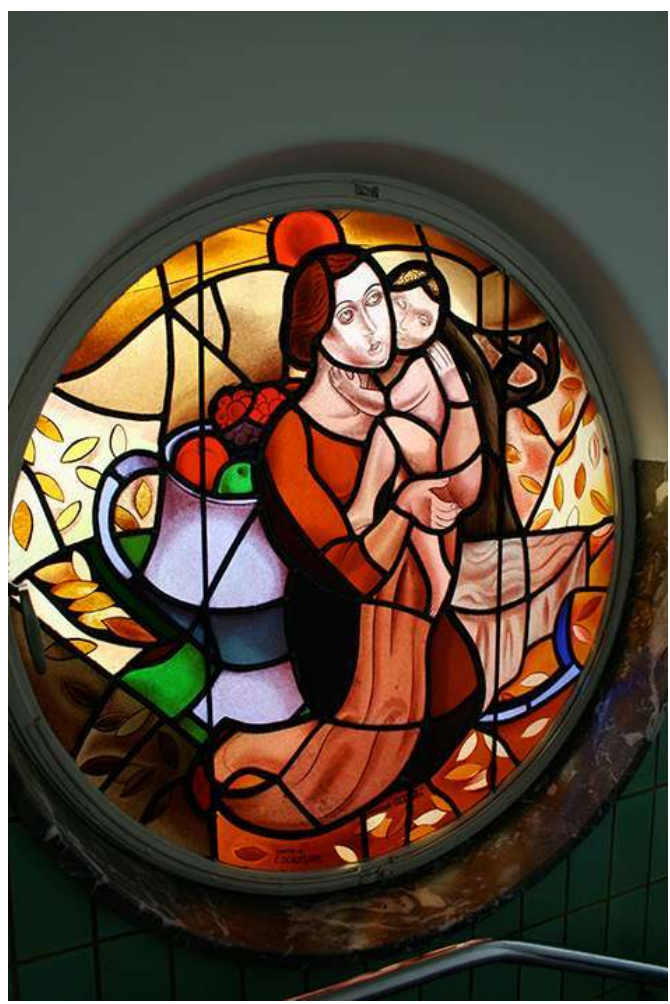

Clinique Jules Seeliger, arch. Joseph Moutschen, 1938-1948. Photographie du vitrail réalisé par Edgar Scauflaire.

Phot. Charlier, Sébastien, 2012. (c) Sébastien Charlier. 
Si l'absence d'archives sur les rapports qu'entretenaient architectes et artistes ne nous permet pas de cerner précisément les stratégies et les processus qui se sont établis lors des différentes phases d'élaboration des bâtiments, les relations qui se dévoilent tant à travers les diverses aventures éditoriales qu'à travers les réalisations témoignent d'un renouveau. Entre l'expérience de L'Euvre artistique et la sortie du premier numéro de L'Équerre, plus de trente années se sont écoulées sans que personne ne parvienne à renouer le dialogue entre architectes et plasticiens. Énoncées par les revues d'avantgarde puis soutenues par les autorités communales, les nouvelles conceptions esthétiques trouvent un lieu d'application dans des infrastructures symbolisant la modernisation de la cité. Ainsi, malgré un contexte économique et politique difficile, la seconde moitié des années 1930 voit la concrétisation d'idéaux qui placent l'intervention de l'artiste au cœur du projet architectural tout en la mettant à la portée du plus grand nombre. Mise entre parenthèses durant la Seconde Guerre mondiale, cette dynamique sera réactivée dès le début des années 1950. Portées par les pouvoirs publics, les grandes agences d'architecture comme EGAU avec la cité modèle de Droixhe (1951-1979) ou le Groupe L'Équerre avec le palais des congrès (1956-1958) s'associeront à des plasticiens locaux et internationaux, donnant un souffle nouveau aux idéaux défendus dans l'entre-deuxguerres.

\section{NOTES}

1. - Prenant pour modèle les cercles artistiques bruxellois des XX puis de La Libre esthétique, créés sous la conduite d'Octave Maus (1856-1919) en réaction à l'académisme, L'CEuvre artistique rassemble, autour de Gustave Serrurier-Bovy, les peintres et graveurs Émile Berchmans (1867-1947), Auguste Donnay (1862-1921) et Armand Rassenfosse (1862-1934), le musicien Sylvain Dupuis (1856-1931) et le professeur en médecine François Henrijean (1860-1932). L'Æuuvre artistique est reconnue pour sa contribution au renouveau des arts à Liège, notamment par l'organisation d'une vaste exposition d'arts appliqués réunissant les travaux d'artistes majeurs de l'avant-garde de la fin du XIX ${ }^{\mathrm{e}}$ siècle parmi lesquels William Morris, Walter Crane ou encore Hector Guimard.

2. - Le manifeste est en outre signé par d'autres personnalités de la littérature belge francophone, à savoir Joseph Duchesne, Géo D’Estemy et Constant de Horion. Voir : Groupe moderne d'art de Liège. Manifeste. Liège : Édition du Groupe moderne d'art et de littérature, janvier 1921.

3. - LIÈGE, René. «Pour servir d'introduction à une des tendances modernes en plastique ». Anthologie, mars 1921, n 1 , p. 1 .

4. - LINZE, Georges. « Généralités sur l'idée artistique moderne ». Ibid., p. 2.

5. - Ibid.

6. - Ibid.

7. - ARON, Paul. La littérature prolétarienne en Belgique francophone depuis 1900. Bruxelles: Labor, 1995, p. 47. Voir aussi BOURGEOIS, Victor. «Le beau par l'utile. Introduction à la conférence ». Anthologie, février-mars 1926, $\mathrm{n}^{\circ}$ 4, p. 5-6.

8. - Annonce publiée dans L'Équerre, novembre 1929, nº 3, p. 1. 
9. - FALISE, Yvon. « Nos conférences : Georges Linze ». L'Équerre, décembre 1929, nº 4, p. 4.

10. - LINZE, Georges. "Notre enquête sur l'Art moderne: l'art contemporain ». L'Équerre, novembre $1929, n^{\circ} 3$, p. 1.

11. - E. « Nos conférences, l'architecture moderne ». L'Équerre, février 1930, n 7, p. 4.

12. - VANTHEMSCHE, Guy. "De mislukking van een vernieuwde economisch politiek in Belgïe voor de tweede wereldoorlog : de OREC (Office de redressement économique) van 1935 tot 1938 ». Revue belge d'histoire contemporaine, 1982, t. XIII, n³ 3-4, p. 339-389.

13. - DESAIVE, Pierre-Yves et PECORARO, Stéphanie. «Le lycée Léonie de Waha ». Dans WARZÉE, Gaëtane (dir.). Le patrimoine moderne et contemporain de Wallonie. De 1792 à 1958. Namur: DGATLP, 1999, p. 246.

14. - Bulletin administratif de la Ville de Liège, 1932, p. 169-171.

15. - Bulletin administratif de la Ville de Liège, 1933, p. 1271.

16. - Bulletin administratif de la Ville de Liège, 1936, p. 188-193.

17. - «Vers la ville saine. Liège. Un lycée, un groupe scolaire, une piscine ». L'Équerre, septembre 1936, n 9, p. 1-2.

18. - « Au lycée Léonie de Waha, architecte Jean Moutschen ». Bâtir, août 1938, nº 69, p. 365.

19. - Signalons qu'au nord, une autre sculpture réalisée par Adelin Salle représente Dionysos.

20. - «L'institut chirurgical de la Société mutuelle des Administrations publiques ». La Maison, avril 1951, n 4 , p. 147.

\section{RÉSUMÉS}

En 1928, des étudiants de l'académie des Beaux-Arts de Liège fondent la revue d'architecture et d'urbanisme L'Équerre. Bien que s'inscrivant tardivement dans la continuité des avant-gardes internationales, le mensuel revendique une vision transversale de l'architecture où la réflexion plastique occupe une place centrale. Dès le départ, elle s'associe à Anthologie, revue imprégnée des théories futuristes et qui diffuse les artistes de l'avant-garde locale. D'abord par le biais de l'édition et des expositions, plasticiens et architectes travaillent ensuite ensemble dans le cadre de projets ambitieux financés par les pouvoirs publics. Cette contribution entend revenir aux racines de cette collaboration en définissant les liens théoriques ou personnels unissant les deux revues. En se concentrant sur les infrastructures publiques, notre analyse montrera comment les artistes et les architectes ont répondu ensemble aux exigences concrètes de la commande en les replaçant dans le contexte social, politique et économique d'une ville belge de seconde importance à la veille de la Seconde Guerre mondiale.

In 1928, students from the Academy of Fine Arts in Liege founded a journal of architecture and town planning called L'Équerre. Although it was something of a latecomer to international avantgarde movements, the monthly magazine set out with a view of architecture that focused on the connections between architecture and art. From the beginning, L'Équerre was associated with Anthology, a magazine marked by the theories of Futurism that published artists of the local avant-garde scene. Starting out in the limited context of magazines and exhibitions, artists and architects then went on to work together on ambitious building projects supported by public money. This article aims look at the roots of this collaboration by examining the theoretical and personal links between the two journals. By focusing on public buildings, our analysis will show what concrete actions artists and architects initiated to respond to architectural commissions 
taking into account the social, political and economic background of a medium-sized Belgian city before the Second World War.

INDEX

Mots-clés : architecture, patrimoine, revue, édition, avant-garde, artistes, infrastructures publiques, Art nouveau, Mouvement moderne, architecture scolaire

Keywords : architecture, heritage, review, edition, avant-garde, artists, public infrastructures, Art nouveau, Modern Movement, school architecture

\section{AUTEUR}

\section{SÉBASTIEN CHARLIER}

Docteur en histoire, art et archéologie, chercheur attaché au service d'histoire de l'art contemporain à l'université de Liège (Belgique) scharlier@ulg.ac.be 